\title{
Electrolyte disorders assessment in solid tumor patients treated with anti-EGFR monoclonal antibodies: a pooled analysis of 25 randomized clinical trials
}

\author{
Qiaoli Wang • Yuexiao Qi • Di Zhang • Caifeng Gong • \\ Anqi Yao • Yi Xiao • Jie Yang • Fuxiang Zhou • \\ Yunfeng Zhou
}

Received: 27 September 2014 / Accepted: 16 December 2014 / Published online: 28 December 2014

(C) The Author(s) 2014. This article is published with open access at Springerlink.com

\begin{abstract}
The role of anti-epithelial growth factor receptor monoclonal antibodies (anti-EGFR MoAbs) in treatmentrelated electrolyte disorders is still controversial. Therefore, we conducted a meta-analysis of published randomized controlled trials (RCTs) to evaluate the incidences and overall risks of all-grade and grade $3 / 4$ electrolyte disorder events. We searched relevant clinical trials from PubMed, EMBASE, and Web of Knowledge databases, meeting proceedings of American Society of Clinical Oncology and the European Society of Medical Oncology, as well as ClinicalTrials.gov. Eligible studies included phases II, III, and IV RCTs. Statistical analysis was performed to calculate the summary incidence, relative risk (RR), and $95 \%$ confidence intervals (CIs) using fixed effects or random effects models based on the heterogeneity of included studies. A total of 16,411 patients from 25 RCTs were included in this meta-analysis. The all-grade incidence of hypomagnesemia related to anti-EGFR MoAbs was $34.0 \%$ (95\% CI 28.0-40.5\%), and that for hypokalemia and hypocalcemia were $14.5 \%$ (95 \% CI $8.2-$ $24.4 \%$ ) and $16.8 \%$ (95\% CI 14.2-19.7\%), respectively. Compared with chemotherapy alone in colorectal cancer,
\end{abstract}

Electronic supplementary material The online version of this article (doi:10.1007/s13277-014-2983-9) contains supplementary material, which is available to authorized users.

Q. Wang · Y. Qi · C. Gong · A. Yao · Y. Xiao · J. Yang · F. Zhou Y. Zhou $(\bowtie)$

Department of Radiation Oncology and Medical Oncology, Zhongnan Hospital of Wuhan University, Hubei Key Laboratory of Tumor Biological Behaviors and Hubei Cancer Clinical Study Center, No. 169 Donghu Road, Wuhan, Hubei 430071, People's Republic of China

e-mail: yfzhouwhu@163.com

\section{Zhang}

Department of Oncology, The First Affiliated Hospital of Soochow University, No. 188 Shizi Street, Suzhou, Jiangsu 215006, People's Republic of China addition of cetuximab increased the risk of grade 3/4 hypomagnesemia and grade $3 / 4$ hypokalemia with RRs of 7.14 (95\% CI 3.13-16.27, $p<0.001)$ and 2.19 (95\% CI 1.14-4.23, $p=0.019$ ). Additionally, colorectal cancer patients in panitumumab cases were more vulnerable to grade $3 / 4$ hypomagnesemia and hypokalemia (RR 18.29, $95 \%$ CI 7.2948.41, $p<0.001$, and RR 3.3, $95 \%$ CI $1.32-8.25, p=0.011$ ). Treatment with anti-EGFR MoAbs is associated with significantly higher risks of electrolyte disorders such as hypomagnesemia, hypomagnesemia, and hypocalcemia, especially in colorectal cancer. Rigorous monitoring and early treatment of electrolyte disorders are proposed.

Keywords Electrolyte disorders · Cetuximab · Panitumumab $\cdot$ Meta-analysis

\section{Introduction}

Epithelial growth factor receptor (EGFR), which is also known as erbB1 or HER1, is the first growth factor receptor to be proposed as a target for anti-cancer therapy [1]. EGFR is a $170-\mathrm{kDa}$ transmembrane protein with an intracellular tyrosine-kinase, which can be overexpressed by a range of different tumors such as colorectal cancer, head and neck cancer, lung cancer, pancreas cancer, and breast cancer [2]. It is crucial in modulating cellular signaling pathways including proliferation, inhibition of apoptosis, angiogenesis, invasion, and metastasis, making it a promising target for anti-cancer agent [3]. At present, anti-EGFR agents mainly include two types: tyrosine-kinase inhibitors (TKI) and monoclonal antibodies (MoAbs) [4]. The MoAbs approved by the US Food and Drug Administration (FDA) include cetuximab (Erbitux ${ }^{\mathrm{TM}}$ ), a chimeric immunoglobulin G1 antibody, in February 2004 and 
panitumumab (Vectibix ${ }^{\mathrm{TM}}$ ), a fully-human immunoglobulin G2 antibody, in September $2006[5,6]$. These agents are still being evaluated in treatment of various advanced malignant diseases such as colorectal cancer, non-small-cell lung cancer (NSCLC), head and neck cancer, and so on. Thus, more applications of MoAbs are expected in the near future.

With respect to side effects, the most specific and frequently toxic effect of anti-EGFR MoAbs is acneiform eruption, skin rash, and other cutaneous events. They have been regarded as typical class adverse events related to MoAbs [7]. However, electrolyte disorders are also common adverse events during anticancer therapy but are often overlooked. If decreased electrolytes have not been managed timely, fetal events like cardiac arrhythmia, coronary artery vasospasm, and sudden cardiac death might take place. What is more, on account of lacking monitoring system, it would be more dangerous in outpatients.

Since the indications for anti-EGFR MoAbs are increasing, it is prerequisite to recognize the patterns of toxic effects such as incidence and relative risk (RR) of electrolyte disorder events and to understand the mechanism of the drug, so that early and essential intervention can be done. To our knowledge, on account of the limited number of patients in trials, there is no clinical trial with a great capacity to explore electrolyte disorders associated with MoAbs agents in detail. Thus, in order to better understand the overall risk of electrolyte disorders, we conducted a meta-analysis of published randomized controlled trials (RCTs) to investigate the incidence and RR of all-grade and grade 3/4 electrolyte disorders during the treatment of anti-EGFR MoAbs (cetuximab or panitumumab).

\section{Methods}

\section{Search strategy}

This study was performed in accordance with Preferred Reporting Items for Systematic Reviews and Meta-Analyses (PRISMA) statement [8]. The first two authors independently conducted a comprehensive literature search of PubMed (January 1, 1966 to June 30, 2014) using the following keywords: "panitumumab," "Vectibix," "ABX-EGF" or "cetuximab," "Erbitux," "C-255," limited by "clinical trial." Then, we manually searched bibliographies of included trials with keywords of "randomized controlled trial" and "adverse events." The same keywords were used to search abstracts and virtual meeting presentations from the American Society of Clinical Oncology (ASCO) and the European Society of Medical Oncology (ESMO) conferences. Information on ongoing registered clinical trials from the National Institutes of Health Web site (http://www.clinicaltrials.gov) was also referenced. The search strategy was also carried out to search the database EMBASE and Web of Knowledge to make sure that no relevant trials were neglected. Only the most recent, complete and full manuscripts from clinical trials were included.

\section{Eligibility criteria}

The principal objective of this study was to determine the incidence and the overall risk of electrolyte disorders associated with anti-EGFR MoAbs. Thus, trials that matched the following criteria were included: (1) participating patients with all solid tumors at baseline; (2) randomized controlled phases II, III, and IV clinical trials; (3) patients were assigned to cetuximab or panitumumab therapy and controls; and (4) data available for the events of electrolyte disorders and sample size for analysis. We excluded studies if they met the following criteria: (1) phase I trials or single arm phase II trials that lack of controls; (2) any meta-analysis, comment, review, and case report; (3) retrospective trials; and (4) trials lack of suitable data of electrolyte disorders.

\section{Data extraction and study quality assessment}

Two authors independently reviewed the full studies and the following information were included into an electronic database: name of first author, year of publication, trial phase, number of patients enrolled and analyzed, patients status, follow-up duration, underlying malignancy, treatment methods, National Cancer Institute's Common Terminology Criteria for Adverse Events (CTCAE) criteria version, and adverse outcomes of events interest (hypomagnesemia, hypokalemia, hypocalcemia, and hyponatremia). We included the exact number of patients who occurred adverse events interest of all-grade and grade 3/4 and number of total patients enrolled in the clinical trials. The study quality was assessed by the same two reviewers independently according to the Jadad score which included randomization, blinding, and withdrawals, ranging from 0 to 5 points [9]. Any discrepancies were resolved by joint review of the manuscript to reach consensus.

\section{Statistical analysis}

We used Comprehensive Meta Analysis program version 2 (Biostat, Engle-wood, NJ, USA) to pool data. The incidence, $\mathrm{RR}$, and their $95 \%$ confidence intervals (CIs) of adverse events were calculated for each study, and the results were compared through both random effects model (Der-Simonian and Laird's method [10]) and fixed effects model (MantelHaenszel method). Statistical heterogeneity among studies was assessed by Cochrane's $Q$ statistic and $I^{2}$ statistic [11]. The $I^{2}$ value provides an estimate of amount of variance across studies derived from heterogeneity rather than chance. If $p$ 
value of Cochrane's $Q$ statistic $<0.1$, the assumption of homogeneity was deemed invalid and a random effects model was reported; otherwise, results from the fixed effect model were reported. RR $>1$ reflects a higher overall risk of adverse events. All $p$ values were two-tailed and were considered statistically significant if $p<0.05$.

Subgroup analysis was performed by tumor type and MoAbs agent category. Sensitivity analysis was based on the weight or quality of the studies to assess the robustness of primary results. Publication bias was evaluated using Begg's and Egger's tests $[12,13]$.

\section{Results}

Literature search results

Seven hundred eighty-four potentially relevant clinical trials with anti-EGFR MoAbs were identified with the search strategy, of which 145 were initially excluded as duplicates (Fig. 1). After a review of the titles and abstracts of the remaining 639 publications, 172 trials were judged as promising articles. These articles were selected and evaluated in greater detail by reviewing the full articles. And, finally, 25
RCTs were considered as highly relevant trials for the metaanalysis.

Study characteristics

Twenty-five RCTs reporting 23,094 patients were identified, among which, 16,411 were actually exposed to the original study. There were 3011 total electrolyte disorder events among these patients (anti-EGFR MoAbs, $n=2161$; controls, $n=850$ ). Table 1 shows the baseline characteristics of each trial. Underlying malignancies included colorectal cancer (11 studies) [14-24], NSCLC (five studies) [25-29], head and neck cancer (three studies) [30-32], oesophageal cancer (three studies) [33-35], pancreatic cancer (one study) [36], gastric cancer (one study) [37], and breast cancer (one study) [38]. National Cancer Institute's Common Terminology Criteria for Adverse Events (CTCAE) criteria, version 2, 3, or 4 were used to evaluate the adverse events in these studies. The differences between the three versions were presented in Supporting Information Table 1 (Online Resource). These trials include 4 phase II [18, 26, 27, 38], 18 phase III [14-17, 19, 21-25, $28-32,34,36,37]$, and 2 phase II/III studies [33, 35], and one study did not report the exact phase [20]. Thirteen studies mentioned follow-up duration, and 21 reported hypomagnesemia events, 16 studies for hypokalemia events, four for
Fig. 1 The literature search process

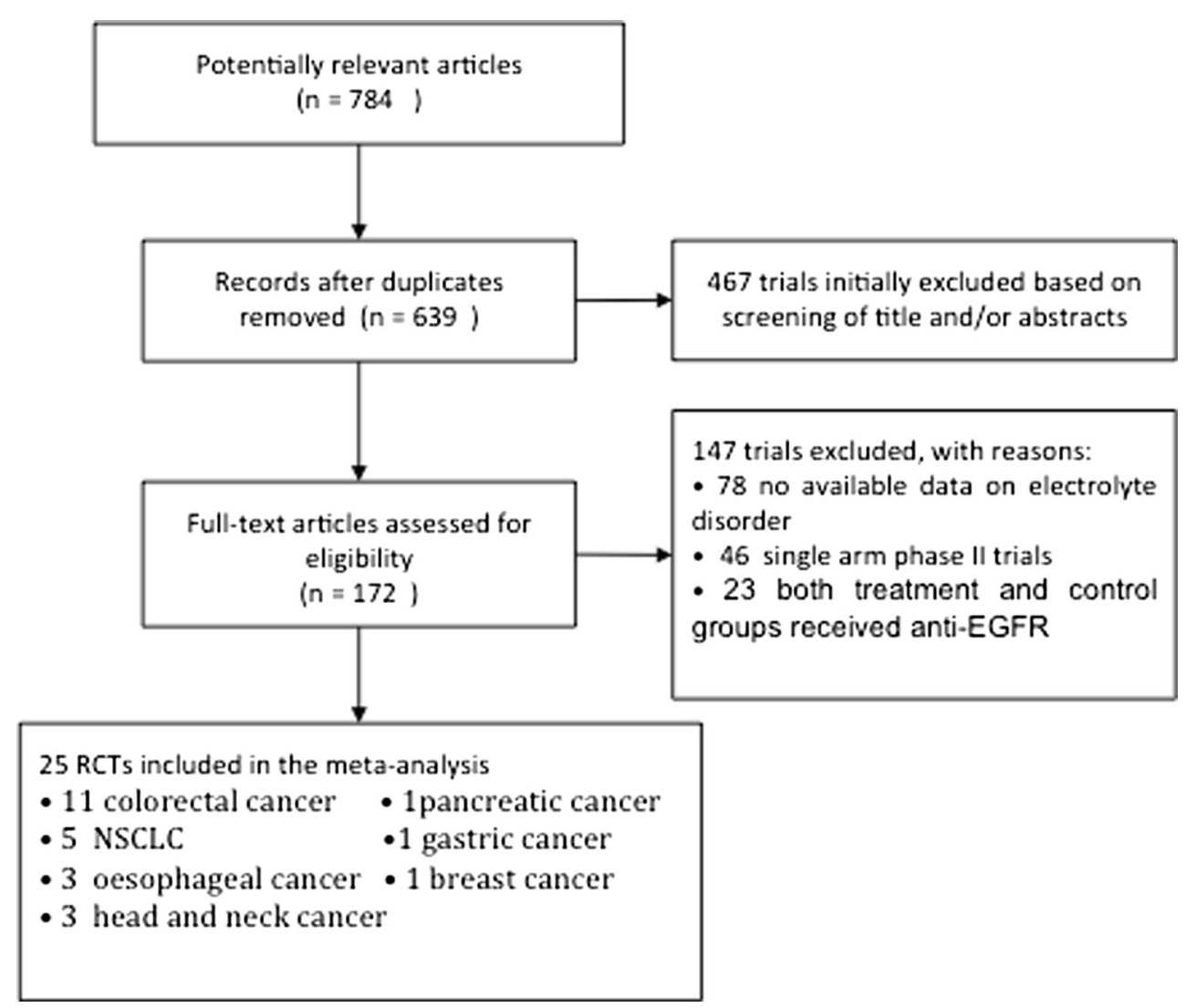




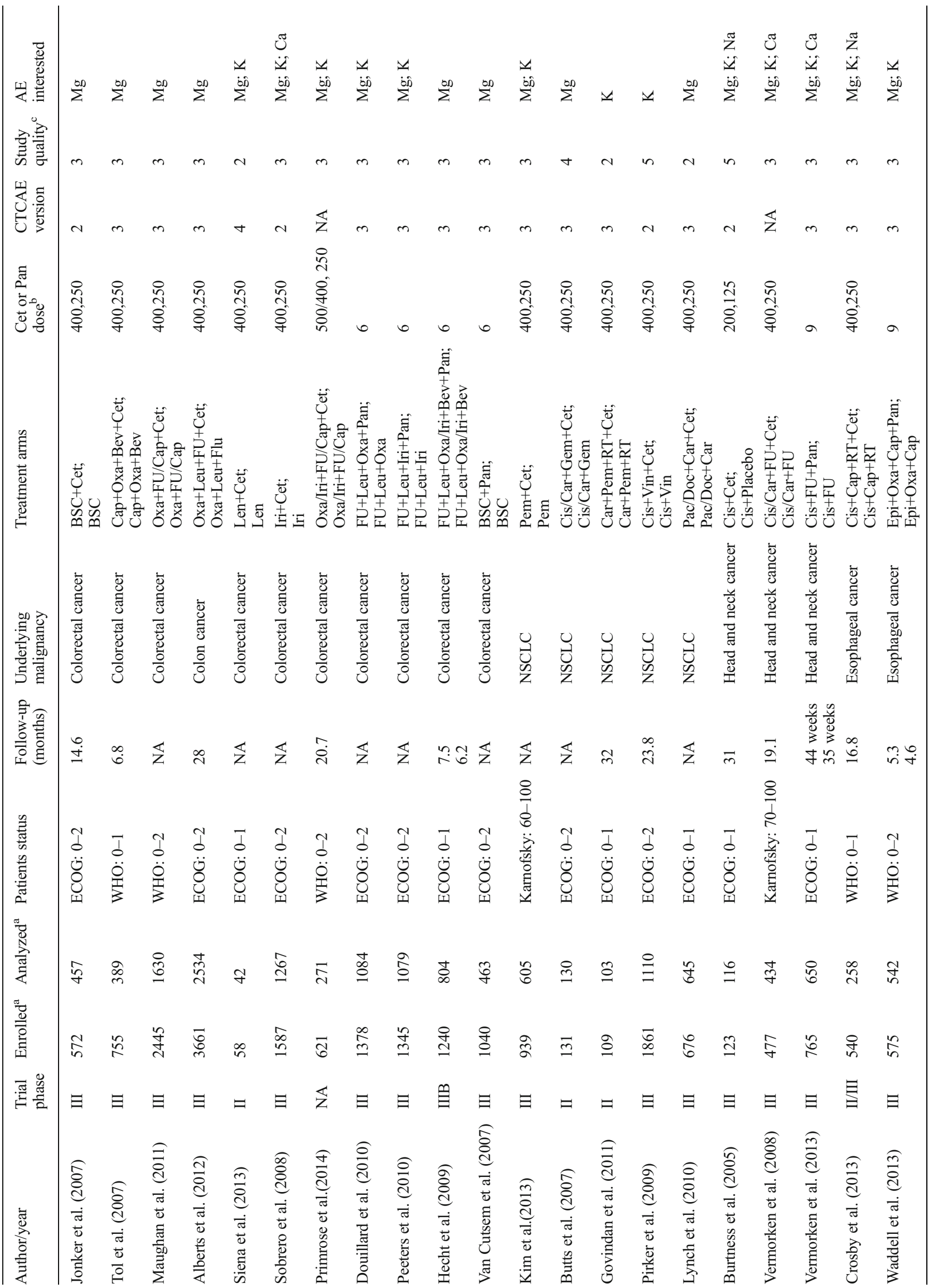


hypocalcemia events, and three for hyponatremia events as shown in Table 1. In all trials, patients were randomly assigned to with or without MoAbs-treated groups, according to Eastern Cooperative Oncology Group performance status or other criteria. Patients enrolled in the MoAbs group received cetuximab $400 \mathrm{mg} / \mathrm{m}^{2}$ at first dose and $250 \mathrm{mg} / \mathrm{m}^{2}$ per week (or $500 \mathrm{mg} / \mathrm{m}^{2}$ every 2 weeks) or panitumumab $6 \mathrm{mg} / \mathrm{kg}$ (or $9 \mathrm{mg} / \mathrm{kg}$ according to the tumor types) on day 1 of each cycle. The overall methodological study quality was generally good and fair with a Jadad mean score of 3.04, ranging from 2 to 5 .

Incidence of electrolyte disorder events

\section{Incidence of hypomagnesemia events}

Twenty RCTs reported grade 3/4, and ten reported all-grade hypomagnesemia events. All-grade hypomagnesemia events were recorded in 879 of 2682 patients in MoAbs-treated group, conferring an incidence of $34.0 \%$ (95\% CI 28.0 $40.5 \%$ ), whereas that in controls was $9.7 \%$ (95\% CI 6.5$14.3 \%$ ) (Table 2), indicating a higher risk of all-grade hypomagnesemia events related to MoAbs (RR 3.37, $95 \% \mathrm{CI}$ 2.41-4.72, $p<0.001$ ) (Online Resource Fig. 1). The incidence of grade 3/4 hypomagnesemia events in MoAbs was also significantly higher than control group (incidence $4.8 \%$, $95 \%$ CI 3.6-6.4\%, vs $0.7 \%$, $95 \%$ CI $0.4-1.2 \%$, $p<0.001$ ), with RR value of 6.10 (95\% CI 4.37-8.52, $p<0.001$ ) (Fig. 2).

Incidence of hypomagnesemia events was then calculated for cetuximab and panitumumab trials separately (Table 2). Of note, among cetuximab trials, incidences of all-grade and grade 3/4 hypomagnesemia events in cetuximab group were approximately three times (incidence $34.9 \%$, $95 \%$ CI 25.9 $45.1 \%$, vs $12.6 \%, 95 \%$ CI $9.0-17.3 \%$ ) and 5.5 times (incidence $4.4 \%, 95 \%$ CI $2.9-6.7 \%$, vs $0.8 \%, 95 \%$ CI $0.6-1.3 \%)$ higher than in controls $(p<0.001$ for both) (Table 2). In panitumumab trials, the effects of hypomagnesemia events were also obvious, all-grade incidence of panitumumab group and control group: $31.8 \%, 95 \% \mathrm{CI}$ $27.9-36.0 \%$, vs $3.8 \%$, $95 \%$ CI $0.7-17.0 \%$; grade $3 / 4$ incidence $5.4 \%$, $95 \%$ CI $3.5-8.3 \%$, vs $0.4 \%$, $95 \%$ CI $0.1-1.9 \%$ (Table 2). Then, the trials included were stratified for underlying malignant disease. The incidence of grade $3 / 4$ hypomagnesemia events related to cetuximab in colorectal cancer trials was $2.9 \%$ (95\% CI 1.7-4.7\%) [14-20]. While the incidence in panitumumab group was higher with $4.6 \%$ (95 \% CI 3.5-6.1\%) (Table 2) [21-24].

\section{Incidence of hypokalemia events}

Sixteen RCTs reported grade 3/4 and six RCTs reported allgrade hypokalemia events. The grade $3 / 4$ events of anti-EGFR MoAbs were noted in 304 of 4543 patients, yielding the 
Table 2 Incidence of grade 3/4 or all-grade hypomagnesemia events with MoAbs according to tumor types and MoAbs agents

\begin{tabular}{|c|c|c|c|c|c|c|c|}
\hline & \multirow[t]{2}{*}{ Groups } & \multirow[t]{2}{*}{ No. } & \multicolumn{2}{|c|}{ No. of grade $3 / 4$ events/total no. } & \multicolumn{2}{|c|}{ Incidence $(95 \% \mathrm{CI})^{\mathrm{a}}$} & \multirow[t]{2}{*}{$p$ value } \\
\hline & & & MoAbs & Control & MoAbs & Control & \\
\hline \multicolumn{8}{|l|}{ Grade $3 / 4$} \\
\hline \multirow[t]{7}{*}{ Cetuximab } & Overall & 14 & $168 / 3798$ & $21 / 3606$ & $4.4(2.9-6.7)$ & $0.8(0.6-1.3)$ & $<0.001$ \\
\hline & Colorectal cancer & 6 & $56 / 2151$ & $5 / 2036$ & $2.9(1.7-4.7)$ & $0.4(0.2-0.8)$ & $<0.001$ \\
\hline & NSCLC & 3 & $33 / 681$ & $4 / 675$ & $3.7(1.2-11.1)$ & $0.7(0.3-1.8)$ & $<0.001$ \\
\hline & Head and neck cancer & 2 & $19 / 277$ & $3 / 273$ & $8.3(3.0-21.0)$ & $1.3(0.5-3.6)$ & 0.001 \\
\hline & Esophageal cancer & 1 & $9 / 129$ & $2 / 129$ & $7.0(3.7-12.9)$ & $1.6(0.4-6.0)$ & 0.031 \\
\hline & Gastric cancer & 1 & $47 / 446$ & $6 / 436$ & $10.5(8.0-13.7)$ & $1.4(0.6-3.0)$ & $<0.001$ \\
\hline & Breast cancer & 1 & $4 / 114$ & $1 / 57$ & $3.5(1.3-9.0)$ & $1.8(0.2-11.4)$ & 0.521 \\
\hline \multirow[t]{4}{*}{ Panitumumab } & Overall & 6 & $138 / 2426$ & $16 / 2420$ & $5.4(3.5-8.3)$ & $0.4(0.1-1.9)$ & $<0.001$ \\
\hline & Colorectal cancer & 4 & $85 / 1825$ & $4 / 1829$ & $4.6(3.5-6.1)$ & $0.3(0.1-0.6)$ & $<0.001$ \\
\hline & Esophageal cancer & 1 & $13 / 276$ & $0 / 266$ & $4.7(2.8-7.9)$ & $0.2(0.0-2.9)$ & $<0.001$ \\
\hline & Head and neck cancer & 1 & $40 / 325$ & $12 / 325$ & $12.3(9.2-16.3)$ & $3.7(2.1-6.4)$ & $<0.001$ \\
\hline Overall & & 20 & $306 / 6224$ & $37 / 6026$ & $4.8(3.6-6.4)$ & $0.7(0.4-1.2)$ & $<0.001$ \\
\hline \multicolumn{8}{|l|}{ All-grade } \\
\hline \multirow[t]{4}{*}{ Cetuximab } & Overall & 7 & $559 / 1659$ & $188 / 1545$ & $34.9(25.9-45.1)$ & $12.6(9.0-17.3)$ & $<0.001$ \\
\hline & Colorectal cancer & 4 & $329 / 857$ & $91 / 754$ & $35.8(24.3-49.1)$ & $12.0(9.0-16.0)$ & $<0.001$ \\
\hline & NSCLC & 2 & $97 / 356$ & $36 / 355$ & $37.0(10.3-75.1)$ & $13.7(2.5-49.7)$ & $<0.001$ \\
\hline & Gastric cancer & 1 & $133 / 446$ & $61 / 436$ & $29.8(25.8-34.2)$ & $14.0(11.0-17.6)$ & $<0.001$ \\
\hline \multirow[t]{3}{*}{ Panitumumab } & Overall & 3 & $320 / 1023$ & $57 / 1010$ & $31.8(27.9-36.0)$ & $3.8(0.7-17.0)$ & $<0.001$ \\
\hline & Colorectal cancer & 2 & $231 / 747$ & $16 / 744$ & $31.9(25.4-39.1)$ & $1.8(0.6-5.4)$ & $<0.001$ \\
\hline & Esophageal cancer & 1 & $89 / 276$ & $41 / 266$ & $32.2(27.0-38.0)$ & $15.4(11.6-20.3)$ & $<0.001$ \\
\hline Overall & & 10 & $879 / 2682$ & $245 / 2555$ & $34.0(28.0-40.5)$ & $9.7(6.5-14.3)$ & $<0.001$ \\
\hline
\end{tabular}

MoAbs monoclonal antibodies, $C I$ confidence interval, NSCLC non-small-cell lung cancer

${ }^{a}$ Calculated using the random-effect model (Comprehensive Meta Analysis 2, Biostat)

incidence of $6.7 \%$ (95\% CI 5.2-8.7\%), whereas in the control group, the incidence was $3.7 \%$ (95 \% CI 2.5-5.4\%) (Online Resource Table 2), implying that addition of antiEGFR MoAbs increased the risk of hypokalemia $(\mathrm{RR}=1.68$, $95 \%$ CI 1.40-2.03; $p<0.001$ ) (Fig. 2). Similarly, incidence of all-grade hypokalemia in MoAbs group ( $14.5 \%, 95 \%$ CI $8.2-$ $24.4 \%)$ was higher than controls $(9.7 \%, 95 \%$ CI 6.0-15.2 \%, $p<0.001$ ) (Online Resource Table 2).

All-grade hypokalemia events with cetuximab occurred in $12.6 \%$ patients and grade $3 / 4$ incidence was $6.1 \%$, both of which were significantly higher than their controls $(p<0.001$ for both) (Online Resource Table 2). In subanalysis of different tumor types, the addition of cetuximab augmented notably the incidence of grade $3 / 4$ events in colorectal cancer (2.7, $95 \%$ CI $0.8-8.3)$ and NSCLC $(5.7,95 \%$ CI 2.9-10.9). For panitumumab treatment group, colorectal cancer patients received an obviously higher incidence of grade $3 / 4$ events than those in controls ( 7.2 vs $2.1 \%, p<0.001)$.

\section{Incidence of hypocalcemia or hyponatremia events}

Four RCTs recorded grade 3/4 and two RCTs recorded allgrade hypocalcemia events. The incidence of all-grade hypocalcemia related to cetuximab was $16.8 \%$ (95\% CI 14.2-19.7\%), while the control was $9.9 \%$ (95\% CI 8.0$12.2 \%$ ). And, grade $3 / 4$ hypocalcemia of cetuximab and panitumumab was $3.8 \%$ compared with $2.0 \%$ (Online Resource Table 3).

Three RCTs noted grade 3/4, and one noted all-grade hyponatremia events associated with anti-EGFR MoAbs treatment (all of them were in cetuximab trials). The overall incidence of grade 3/4 and all-grade events was $7.8 \%(95 \%$ CI $2.1-25.0 \%)$ and $9.4 \%(95 \%$ CI 7.0-12.5\%), respectively. However, no significant difference was found $(p>0.05$ for both) (Online Resource Table 4).

Relative risk of grade 3/4 electrolyte disorder events

\section{Relative risk of grade 3/4 hypomagnesemia events}

As an exploratory analysis, patients were stratified according to anti-EGFR MoAbs (cetuximab or panitumumab) and underlying malignant disease. Studies of cetuximab showed that 14 RCTs were available to calculate the RR of grade $3 / 4$ hypomagnesemia events. Events treated with or without cetuximab were 168/3798 and 21/3606 (RR 6.23, $95 \%$ CI 
Fig. 2 The overall relative risk of different grade $3 / 4$ electrolyte disorder events associated with MoAbs

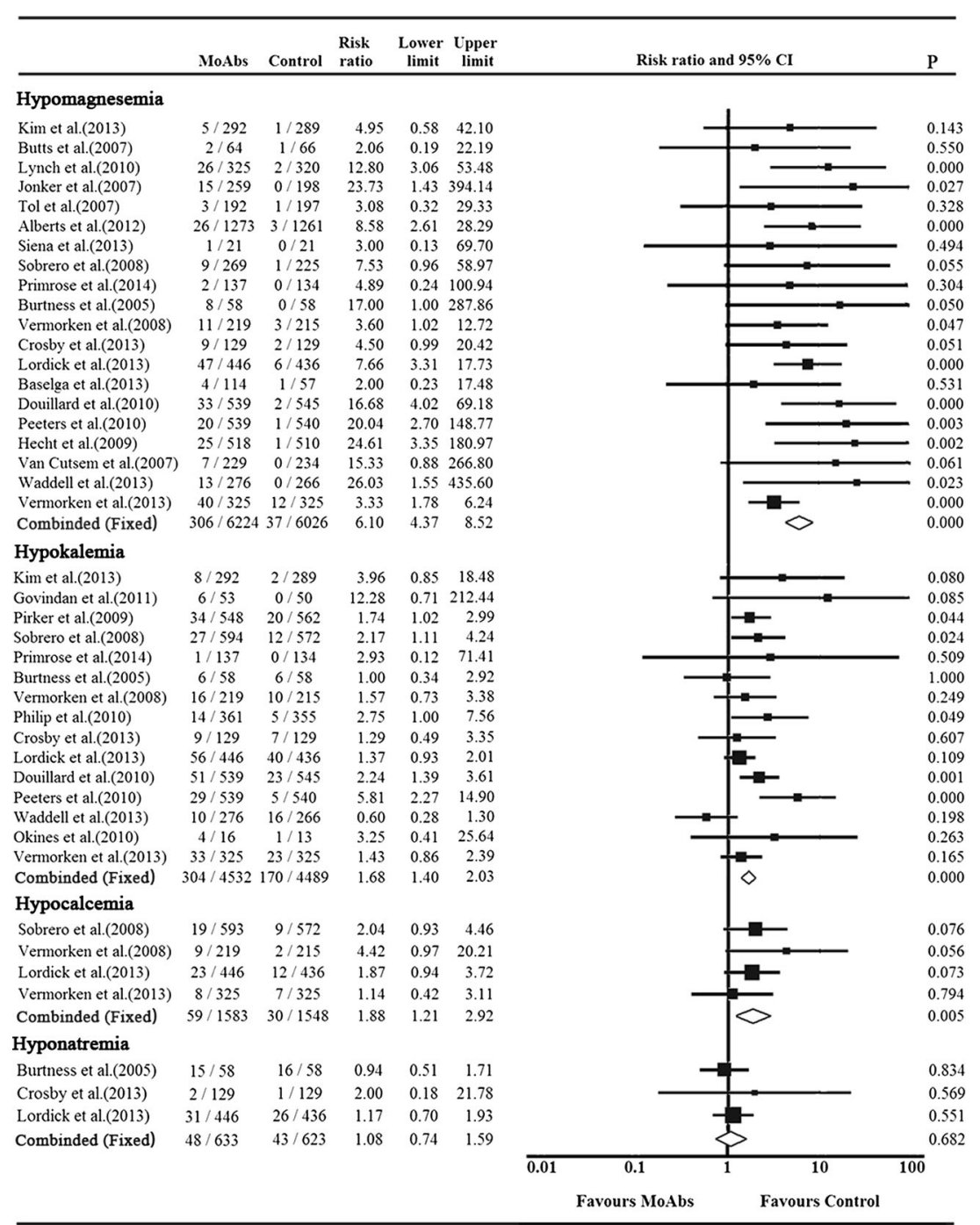

4.01-9.66, $p<0.001)$ and the $p$ value of Cochrane's $Q$ statistic was $0.93\left(I^{2}=0\right)$, justifying the use of the fixed effect model (Mantel-Haenszel) (Fig. 3). Six RCTs of panitumumab contained 138 grade 3/4 hypomagnesemia events in 2426 patients compared with 16 events in 2420 patients in control (RR 11.18, 95 \% CI 4.20-29.80, $p<0.001$ ) (Fig. 3) using random effect model. The heterogeneity existed $(p=0.072$, $I^{2}=50.7 \%$ ), and sensitivity analysis was conducted. It seemed that when we omitted the study conducted by Vermorken et al., the heterogeneity decreased to $0 \%(p=0.997)$, though the result was precarious with RR of 19.42 (95 \% CI 7.9247.63, $p<0.001$ ) (Fig. 3).

When stratifying for underlying cancers, we noted the RR of grade 3/4 hypomagnesemia events varied either in cetuximab trials $(p<0.001)$ or panitumumab trials $(p=0.004$, data not shown). Subanalysis showed that colorectal cancer patients had the highest RR: 7.14 of cetuximab (95\% CI $3.13-16.27, p<0.001)$ and 18.79 of panitumumab (95\% CI 7.29-48.41, $p<0.001$ ) (Fig. 3). Significant statistical differences were also observed in NSCLC $(\mathrm{RR}=7.02,95 \%$ CI 2.43-20.34, $p<0.001)$, head and neck cancer $(4.66,95 \%$ CI 1.47-14.76, $p=0.009)$ in cetuximab trials, as well as noncolorectal cancer in panitumumab trials $(3.67,95 \%$ CI $1.99-$ 6.77, $p<0.001$ ) (Fig. 3).

\section{Relative risk of grade 3/4 hypokalemia events}

Upon stratification by MoAbs agents, we observed that the RR of grade 3/4 hypokalemia events was 1.64 (95 \% CI 1.29$2.08, p<0.001)$ for cetuximab-based regimens and $1.86(95 \%$ CI $0.95-3.61, p=0.069)$ for panitumumab-based regimens (Fig. 4). No heterogeneity was detected among ten cetuximab trials ( $p=0.642, I^{2}=0$ ). As some trials had a wide variation in confidence intervals in panitumumab subgroup, which could decline the precision of pooled results, thus a sensitivity analysis was conducted to examine the stability and reliability of the overall RRs by sequential omission of individual studies. When the study results reported by Waddell et al. was 
Fig. 3 Relative risk of grade 3/4 hypomagnesemia events stratified by tumor types and MoAbs agents

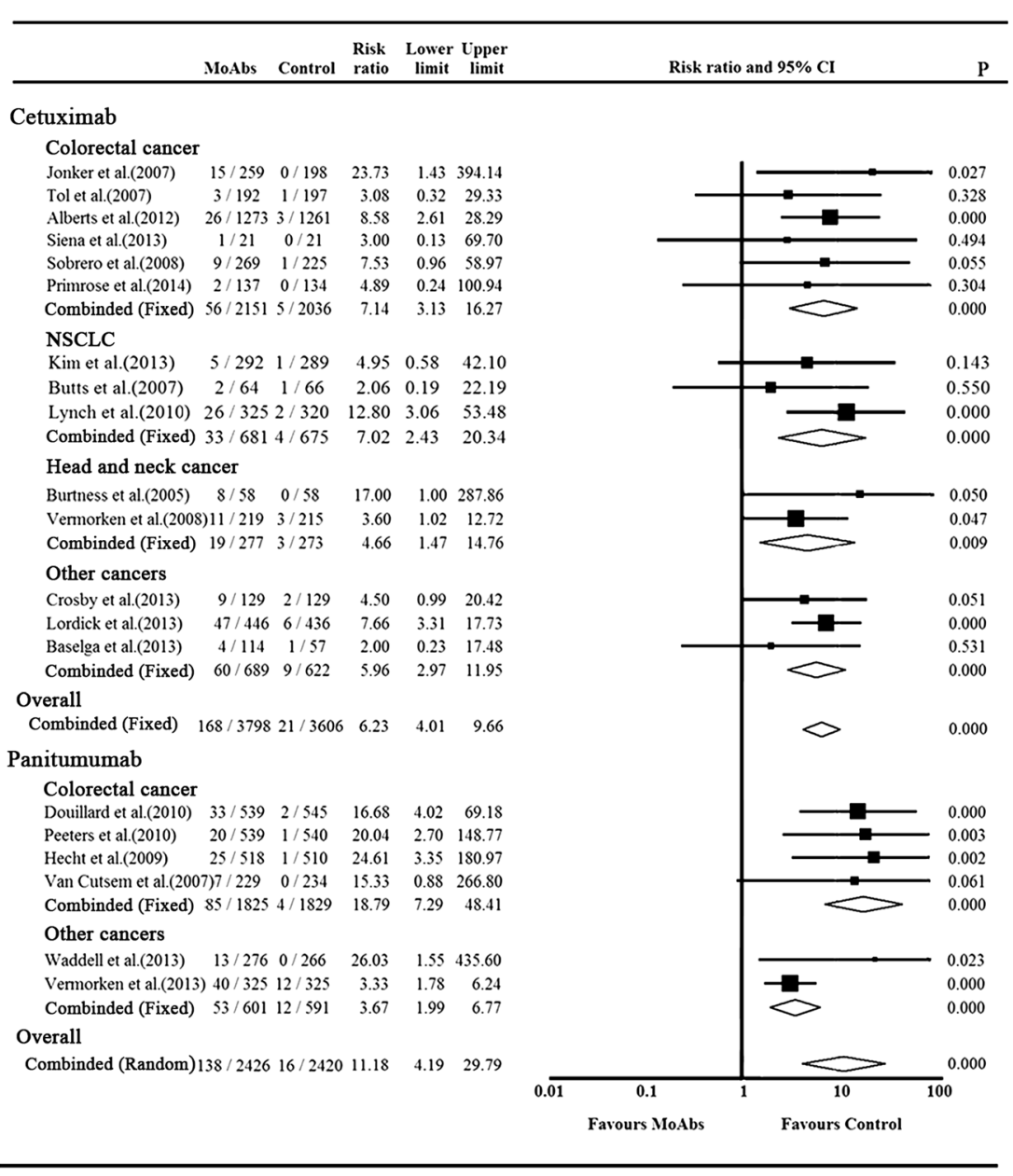

omitted [34], which seemed to explain the heterogeneity among those studies, the RR value turned into 2.40 (95\% CI $1.36-4.24)$ and the heterogeneity among the remaining studies decrease to $56 \%(p=0.076)$.

RRs of grade $3 / 4$ hypokalemia in cetuximab trials were significantly different between tumor types $(p<0.001)$ but were similar in panitumumab trials $(p=0.239$, data not shown). Colorectal cancer patients presented the lower level of RR in cetuximab-based therapy $(\mathrm{RR}=2.19,95 \%$ CI $1.14-4.23, p=$ $0.019)$ than in panitumumab-based therapy $(\mathrm{RR}=3.30,95 \% \mathrm{CI}$ 1.32-8.25, $p=0.011$ ) (Fig. 4). And, significant statistical difference was also observed in NSCLC patients in cetuximab trials $(p=0.006)$. All the subgroup analysis were judged to use fixed effect model due to the $p$ values of Cochrane's $Q$ statistic of $>0.1$, except those colorectal cancer patients treated with panitumumab with the $p$ value of $0.077\left(I^{2}=68.0 \%\right)$, which was calculated using random effect model.

\section{Relative risk of grade 3/4 hypocalcemia or hyponatremia events}

Three RCTs reported grade 3/4 hypocalcemia related to cetuximab, and only one RCT recorded the events with panitumumab. Patients with cetuximab-based therapy had a significantly higher risk of electrolyte disorders $(\mathrm{RR}=2.12$, $95 \%$ CI 1.30-3.45, $p=0.003$ ) (Online Resource Fig. 2), whereas panitumumab did not increase this events as reported $(\mathrm{RR}=1.14,95 \%$ CI 0.42-3.12, $p=0.794$ ) (Online Resource Fig. 2).

The analysis of grade 3/4 hyponatremia events showed that RR in cetuximab trials was $1.08(95 \%$ CI 0.74-1.59, $p=0.682$ ) (Online Resource Fig. 3), but no one reported the events of panitumumab. Due to a lack of sufficient studies, subgroup analysis of different tumor types was not conducted.

Publication bias

The publication bias was performed in the pooling analysis of risk of grade 3/4 hypomagnesemia events and hypokalemia events associated with cetuximab due to the fact that the included studies were more than ten. Evidence of publication bias was not detected by either Begg's test or Egger's test (RR of hypomagnesemia event: Begg's test $p=0.584$, Egger's test $p=0.441$, RR of hypokalemia event: Begg's test $p=0.152$, Egger's test $p=0.074$, respectively) 
Fig. 4 Relative risk of grade 3/4 hypokalemia events stratified by tumor types and MoAbs agents

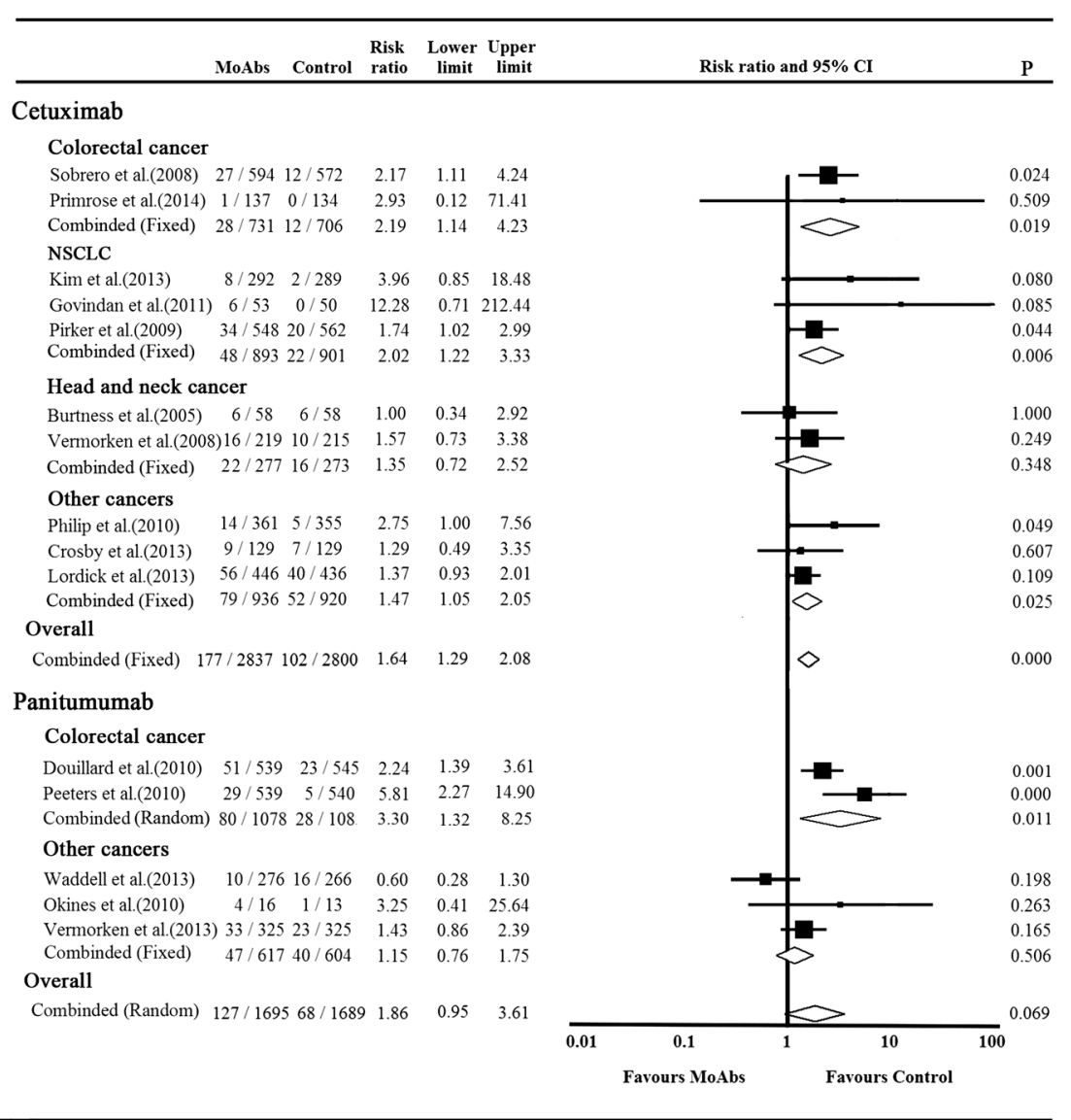

\section{Discussion}

Studies that investigated the toxicity of anti-EGFR MoAbs mainly focused on the common adverse events, such as skin rash [39], hematologic toxicity [40], and specific electrolyte disturbance like hypomagnesemia [41]. To our best knowledge, seldom study has synthetically studied the incidence and risk of allgrade and grade $3 / 4$ electrolyte disorders of cetuximab- or panitumumab-related therapy. As EGFR can be overexpressed in a wide range of tumors and it correlates with poor survival and cancer progression, inhibition of EGFR signaling pathway will be a promising therapeutic target [42]. However, cetuximab and panitumumab both bind with high affinity to human EGFR and hence could reinforce the cytotoxic effects of conventional chemotherapy or chemoradiotherapy [43]. Therefore, the overall benefits of anti-EGFR MoAbs remain to be confirmed. Electrolyte disorders are quite common in overwhelming majority of cancer patients and may result in serious adverse events. Adequate recognition and management of electrolyte disorders is important for those patients who receive anti-EGFR MoAbs therapy. However, the relationship between grade 3/4 electrolyte disorder events and MoAbs-based therapy is difficult to evaluate in individual RCTs for a lack of enough patients.

In our study, data from 25 RCTs was pooled to overcome this limitation and the results demonstrated that therapy with
anti-EGFR MoAbs can dramatically increased the risk of grade $3 / 4$ hypomagnesemia events $(\mathrm{RR}=6.10,95 \% \mathrm{CI}$ $4.37-8,52, p<0.001$; incidence compared with controls: 4.8 vs $0.7 \%$ ). And, all-grade events reached as high as $34.0 \%$ in MoAbs-treated group, compared with $9.7 \%$ in controls $(p<0.001)$. Colorectal cancer patients had the highest risk of grade $3 / 4$ hypomagnesemia events among cancer patients (cetuximab: $\mathrm{RR}=7.14$; panitumumab: $\mathrm{RR}=18.79$ ). Meanwhile, MoAbs also obviously increased RR of grade 3/ 4 hypokalemia and hypocalcemia events with the value of 1.68 and 1.88 , respectively. Interestingly, colorectal cancer patients were also more prone to have grade $3 / 4$ hypokalemia events than others (cetuximab: $R R=2.19$; panitumumab: $\mathrm{RR}=3.30$ ), which was similar with that of grade 3/4 hypomagnesemia events. However, no obviously higher risk of hyponatremia events related to MoAbs was discovered in our study. In brief, the risk of electrolyte disorder events was dramatically increased if the anti-EGFR therapy was added. Therefore, more attention should be paid to the electrolyte disorders when patients treated with anti-EGFR MoAb agents alone or combined with chemotherapy, whereas the mechanism behind these toxicities has not yet been well identified.

Recent studies tend to suggest that a new $\mathrm{Mg}^{2+}$ permeable channel TRPM6 (transient receptor potential cation channel, subfamily $\mathrm{M}$, member 6) and TRPM7 were involved in 
transepithelial $\mathrm{Mg}^{2+}$ transport in the distal convoluted tubule [44]. And, pro-EGF and TRPM6 were both predominantly expressed in distal convoluted tubule, which was the main site of active renal $\mathrm{Mg}^{2+}$ reabsorption. However, the stimulatory effect of EGF on TRPM6 activity could be diminished by preincubation of cetuximab, an EGFR blockade, thus affecting $\mathrm{Mg}^{2+}$ transport and leading to hypomagnesemia [45]. Hypokalemia may relate to hypomagnesemia through TRPM6 [46]. Increasing potassium is required to repair Na-K-ATPase due to the magnesium deficiency. Then, over-intracellular transport of potassium could result in hypokalemia. Thus, on the contrary, sodium is mainly unaltered due to the extracellular transport. Human TRPM6 also give rise to hypomagnesemia with secondary hypocalcemia (HSH) [47]. Although the mechanism responsible for development of hypocalcemia is unclear, several explanations such as end-organ unresponsiveness to parathyroid hormone (PTH), altered release of PTH, and impaired formation of 1,25-dihydroxy vitamin D3 are offered. The exact pathogenesis of anti-EGFR MoAbs associated electrolyte disorders remains to be elucidated.

In particular, the grade $3 / 4$ electrolyte disorders risk is higher in colorectal cancer patients yet not the incidence, when compared with other tumors. The differences in the results are still unknown. However, it has to be noted that most trials of other tumors had implemented a treatment with cisplatin as chemotherapy, a well-known harmful agent to renal convoluted tubules. And, it could aggravate the renal function and result in an extensive electrolyte disorders. On the other hand, 70-75\% colorectal cancer patients overexpress EGFR [48], more than other cancer patients like NSCLC with $60 \%$ [49]. Thus, this anti-EGFR treatment might obviously raise the risks of electrolyte disorders with MoAbs as explained previously.

To note that patients received panitumumab therapy were more inclined to have severe electrolyte disorders than cetuximab. The possible reason can be the absolutely high affinity to human EGFR of panitumumab that reinforced its adverse events [43]. Moreover, the longer half-life of panitumumab than cetuximab ( 7.5 vs 4.7 days) rises the possibility that the different pharmacokinetics of the two MoAbs somehow matters.

For the high risk of severe hypomagnesemia events, most studies mainly focus on the magnesium level in serum. The RR of grade 3/4 hypomagnesemia events associated with cetuximab reported by Petrelli et al. was 9.81 [41], higher than 8.6 from Chen et al. [50] and 6.23 from our study. And, the RR related with panitumumab in Petreli's study was 11.68 , which was similar to the result of our result. The controversial differences may be rooted in different qualities of clinical trials included and some updated trials that were not included in the previous studies.
However, despite the size of this meta-analysis, several limitations are still worth considering. First, these studies were conducted at major academic institutions among patients in hospital and with adequate major organ function, which may neglect outpatients and patients with organ dysfunction, thus could not reflect the general patient population. Second, the process of recording the electrolyte disorder events varies in each clinical trial since these events were not the primary end point of their trials. Therefore, a bias of reported incidence rates emerges. Publication bias of risk of hypomagnesemia and hypokalemia analysis in cetuximab setting was not detected according to Begg's and Egger's test. However, it should be interpreted with caution, as these methodologies are not fully bias-free. Since the publication bias test was only performed on the cetuximab setting which included more than ten studies, there might be some evidences of publication bias in the remaining analyses. Third, this is a meta-analysis at study level, so we can not solve the confounding factors that inherent in those included trials due to the inadequate control and thus could result in potential bias and toward exaggeration or underestimation of risk estimates, such as different chemotherapy exposures, different CTCAE versions and failure to follow-up cases, all of which may lead to the existence of heterogeneity in the incidence of adverse events. Finally, the number of trials that recorded hypocalcemia and hyponatremia events was limited. It is likely that significant differences in hypocalcemia events between treatment arm and controls in panitumumab trials would arise if more studies were available. Nonetheless, all efforts have been made to contain all related trials and all of them are well-conducted. Majority of them are multicenter randomized phase III trials (many are registered studies). This meta-analysis thus pooled a limited but robust "core" of clinical data to draw a final unequivocal result.

\section{Conclusion}

Our results indicated that incidences of electrolyte disorders were obviously elevated with anti-EGFR MoAbs therapy, especially in colorectal cancer patients. Addition of antiEGFR MoAbs would dramatically increase risk of hypomagnesemia events, as well as hypokalemia and hypocalcemia. Panitumumab seemed to have a higher risk in causing severe electrolyte disorders than cetuximab. Among different cancers, colorectal cancer patients receiving anti-EGFR MoAbs treatment showed the highest risk of electrolyte disorders compared with their controls. However, majority of patients with electrolyte disorders are asymptomatic, although symptoms such as fatigue, muscular cramps, and cardiac arrhythmias could be associated with electrolyte disorders. Given its high incidence and risk, rigorous monitoring and early treatment of electrolyte disorders are proposed. 
Conflict of interest None

Funding None

Open Access This article is distributed under the terms of the Creative Commons Attribution License which permits any use, distribution, and reproduction in any medium, provided the original author(s) and the source are credited.

\section{References}

1. Ciardiello F, Tortora G. EGFR antagonists in cancer treatment. N Engl J Med. 2008;358:1160-74.

2. Harris M. Monoclonal antibodies as therapeutic agents for cancer. Lancet Oncol. 2004;5:292-302.

3. Pal SK, Pegram M. Epidermal growth factor receptor and signal transduction: potential targets for anti-cancer therapy. Anticancer Drugs. 2005;16:483-94.

4. Baselga J. The EGFR as a target for anticancer therapy-focus on cetuximab. Eur J Cancer. 2001;37(Supplement 4):16-22.

5. Cunningham D, Humblet Y, Siena S, Khayat D, Bleiberg H, Santoro A, et al. Cetuximab monotherapy and cetuximab plus irinotecan in irinotecan-refractory metastatic colorectal cancer. N Engl J Med. 2004;351:337-45.

6. Giusti RM, Shastri KA, Cohen MH, Keegan P, Pazdur R. FDA drug approval summary: panitumumab (Vectibix). Oncologist. 2007;12: 577-83.

7. Gatzemeier U, von Pawel J, Vynnychenko I, Zatloukal P, de Marinis $\mathrm{F}$, Eberhardt WE, et al. First-cycle rash and survival in patients with advanced non-small-cell lung cancer receiving cetuximab in combination with first-line chemotherapy: a subgroup analysis of data from the FLEX phase 3 study. Lancet Oncol. 2011;12:30-7.

8. Moher D, Liberati A, Tetzlaff J, Altman DG. Preferred reporting items for systematic reviews and meta-analyses: the PRISMA statement. Ann Intern Med. 2009;151:264-9.

9. Jiang N, Wang B, Chen A, Dong F, Yu B. Operative versus nonoperative treatment for acute Achilles tendon rupture: a meta-analysis based on current evidence. Int Orthop. 2012;36:765-73.

10. DerSimonian R, Laird N. Meta-analysis in clinical trials. Control Clin Trials. 1986;7:177-88.

11. Higgins JPT, Thompson SG, Deeks JJ, Altman DG. Measuring inconsistency in meta-analyses. Br Med J. 2003;327:557-60.

12. Begg CB, Mazumdar M. Operating characteristics of a rank correlation test for publication bias. Biometrics. 1994;50:1088-101.

13. Egger M, Smith GD, Schneider M, Minder C. Bias in meta-analysis detected by a simple, graphical test. BMJ. 1997;315:629-34.

14. Jonker DJ, O'Callaghan CJ, Karapetis CS, Zalcberg JR, Tu D, Au HJ, et al. Cetuximab for the treatment of colorectal cancer. N Engl J Med. 2007;357:2040-8.

15. Tol J, Koopman M, Rodenburg CJ, Cats A, Creemers GJ, Schrama JG, et al. A randomised phase III study on capecitabine, oxaliplatin and bevacizumab with or without cetuximab in first-line advanced colorectal cancer, the CAIRO2 study of the Dutch Colorectal Cancer Group (DCCG). An interim analysis of toxicity. Ann Oncol. 2008;19:734-8.

16. Maughan TS, Adams RA, Smith CG, Meade AM, Seymour MT, Wilson RH, et al. Addition of cetuximab to oxaliplatin-based firstline combination chemotherapy for treatment of advanced colorectal cancer: results of the randomised phase 3 MRC COIN trial. Lancet. 2011;377:2103-14.
17. Alberts SR, Sargent DJ, Nair S, Mahoney MR, Mooney M, Thibodeau SN, et al. Effect of oxaliplatin, fluorouracil, and leucovorin with or without cetuximab on survival among patients with resected stage III colon cancer: a randomized trial. JAMA. 2012;307:1383-93.

18. Siena S, Van Cutsem E, Li M, Jungnelius U, Romano A, Beck R, et al. Phase II open-label study to assess efficacy and safety of lenalidomide in combination with cetuximab in KRAS-mutant metastatic colorectal cancer. PLoS One. 2013;8:e62264.

19. Sobrero AF, Maurel J, Fehrenbacher L, Scheithauer W, Abubakr YA, Lutz MP, et al. EPIC: phase III trial of cetuximab plus irinotecan after fluoropyrimidine and oxaliplatin failure in patients with metastatic colorectal cancer. J Clin Oncol. 2008;26:2311-9.

20. Primrose J, Falk S, Finch-Jones M, Valle J, O'Reilly D, Siriwardena A, et al. Systemic chemotherapy with or without cetuximab in patients with resectable colorectal liver metastasis: the New EPOC randomised controlled trial. Lancet Oncol. 2014;15:601-11.

21. Douillard JY, Siena S, Cassidy J, Tabernero J, Burkes R, Barugel M, et al. Randomized, phase III trial of panitumumab with infusional fluorouracil, leucovorin, and oxaliplatin (FOLFOX4) versus FOLFOX4 alone as first-line treatment in patients with previously untreated metastatic colorectal cancer: the PRIME study. J Clin Oncol. 2010;28:4697-705.

22. Peeters M, Price TJ, Cervantes A, Sobrero AF, Ducreux M, Hotko Y, et al. Randomized phase III study of panitumumab with fluorouracil, leucovorin, and irinotecan (FOLFIRI) compared with FOLFIRI alone as second-line treatment in patients with metastatic colorectal cancer. J Clin Oncol. 2010;28:4706-13.

23. Hecht JR, Mitchell E, Chidiac T, Scroggin C, Hagenstad C, Spigel D, et al. A randomized phase IIIB trial of chemotherapy, bevacizumab, and panitumumab compared with chemotherapy and bevacizumab alone for metastatic colorectal cancer. J Clin Oncol. 2009;27:672-80.

24. Van Cutsem E, Peeters M, Siena S, Humblet Y, Hendlisz A, Neyns B, et al. Open-label phase III trial of panitumumab plus best supportive care compared with best supportive care alone in patients with chemotherapy-refractory metastatic colorectal cancer. J Clin Oncol. 2007;25:1658-64.

25. Kim ES, Neubauer M, Cohn A, Schwartzberg L, Garbo L, Caton J, et al. Docetaxel or pemetrexed with or without cetuximab in recurrent or progressive non-small-cell lung cancer after platinum-based therapy: a phase 3, open-label, randomised trial. Lancet Oncol. 2013;14: 1326-36.

26. Butts CA, Bodkin D, Middleman EL, Englund CW, Ellison D, Alam $\mathrm{Y}$, et al. Randomized phase II study of gemcitabine plus cisplatin or carboplatin [corrected], with or without cetuximab, as first-line therapy for patients with advanced or metastatic non small-cell lung cancer. J Clin Oncol. 2007;25:5777-84.

27. Govindan R, Bogart J, Stinchcombe T, Wang X, Hodgson L, Kratzke R, et al. Randomized phase II study of pemetrexed, carboplatin, and thoracic radiation with or without cetuximab in patients with locally advanced unresectable non-small-cell lung cancer: Cancer and Leukemia Group B trial 30407. J Clin Oncol. 2011;29:3120-5.

28. Pirker R, Pereira JR, Szczesna A, von Pawel J, Krzakowski M, Ramlau R, et al. Cetuximab plus chemotherapy in patients with advanced non-small-cell lung cancer (FLEX): an open-label randomised phase III trial. Lancet. 2009;373:1525-31.

29. Lynch TJ, Patel T, Dreisbach L, McCleod M, Heim WJ, Hermann $\mathrm{RC}$, et al. Cetuximab and first-line taxane/carboplatin chemotherapy in advanced non-small-cell lung cancer: results of the randomized multicenter phase III trial BMS099. J Clin Oncol. 2010;28:911-7.

30. Burtness B, Goldwasser MA, Flood W, Mattar B, Forastiere AA, Eastern Cooperative Oncology G. Phase III randomized trial of cisplatin plus placebo compared with cisplatin plus cetuximab in 
metastatic/recurrent head and neck cancer: an Eastern Cooperative Oncology Group study. J Clin Oncol. 2005;23:8646-54.

31. Vermorken JB, Mesia R, Rivera F, Remenar E, Kawecki A, Rottey S, et al. Platinum-based chemotherapy plus cetuximab in head and neck cancer. N Engl J Med. 2008;359:1116-27.

32. Vermorken JB, Stohlmacher-Williams J, Davidenko I, Licitra L, Winquist E, Villanueva C, et al. Cisplatin and fluorouracil with or without panitumumab in patients with recurrent or metastatic squamous-cell carcinoma of the head and neck (SPECTRUM): an open-label phase 3 randomised trial. Lancet Oncol. 2013;14:697-710.

33. Crosby T, Hurt CN, Falk S, Gollins S, Mukherjee S, Staffurth J, et al. Chemoradiotherapy with or without cetuximab in patients with oesophageal cancer (SCOPE1): a multicentre, phase $2 / 3$ randomised trial. Lancet Oncol. 2013;14:627-37.

34. Waddell T, Chau I, Cunningham D, Gonzalez D, Okines AF, Okines $\mathrm{C}$, et al. Epirubicin, oxaliplatin, and capecitabine with or without panitumumab for patients with previously untreated advanced oesophagogastric cancer (REAL3): a randomised, open-label phase 3 trial. Lancet Oncol. 2013;14:481-9.

35. Okines AF, Ashley SE, Cunningham D, Oates J, Turner A, Webb J, et al. Epirubicin, oxaliplatin, and capecitabine with or without panitumumab for advanced esophagogastric cancer: dose-finding study for the prospective multicenter, randomized, phase II/III REAL-3 trial. J Clin Oncol. 2010;28:3945-50.

36. Philip PA, Benedetti J, Corless CL, Wong R, O'Reilly EM, Flynn PJ, et al. Phase III study comparing gemcitabine plus cetuximab versus gemcitabine in patients with advanced pancreatic adenocarcinoma: Southwest Oncology Group-directed intergroup trial S0205. J Clin Oncol. 2010;28:3605-10.

37. Lordick F, Kang YK, Chung HC, Salman P, Oh SC, Bodoky $\mathrm{G}$, et al. Capecitabine and cisplatin with or without cetuximab for patients with previously untreated advanced gastric cancer (EXPAND): a randomised, open-label phase 3 trial. Lancet Oncol. 2013;14:490-9.

38. Baselga J, Gomez P, Greil R, Braga S, Climent MA, Wardley AM, et al. Randomized phase II study of the anti-epidermal growth factor receptor monoclonal antibody cetuximab with cisplatin versus cisplatin alone in patients with metastatic triple-negative breast cancer. $\mathrm{J}$ Clin Oncol. 2013;31:2586-92.
39. Balagula Y, Wu S, Su X, Lacouture ME. The effect of cytotoxic chemotherapy on the risk of high-grade acneiform rash to cetuximab in cancer patients: a meta-analysis. Ann Oncol. 2011;22:2366-74.

40. Cui R, Chu L, Liu ZQ, Xiao YY, Zhu XL, Chen YJ et al. Hematologic toxicity assessment in solid tumor patients treated with cetuximab: a pooled analysis of 18 randomized controlled trials. Int $\mathrm{J}$ Cancer. 2014

41. Petrelli F, Borgonovo K, Cabiddu M, Ghilardi M, Barni S. Risk of anti-EGFR monoclonal antibody-related hypomagnesemia: systematic review and pooled analysis of randomized studies. Expert Opin Drug Saf. 2012;11 Suppl 1:S9-19.

42. Mendelsohn J, Baselga J. The EGF receptor family as targets for cancer therapy. Oncogene. 2000;19:6550-65.

43. Bruns CJ, Harbison MT, Davis DW, Portera CA, Tsan R, McConkey DJ, et al. Epidermal growth factor receptor blockade with C225 plus gemcitabine results in regression of human pancreatic carcinoma growing orthotopically in nude mice by antiangiogenic mechanisms. Clin Cancer Res. 2000;6:1936-48.

44. Schlingmann KP, Weber S, Peters M, Niemann Nejsum L, Vitzthum $\mathrm{H}$, Klingel $\mathrm{K}$, et al. Hypomagnesemia with secondary hypocalcemia is caused by mutations in TRPM6, a new member of the TRPM gene family. Nat Genet. 2002;31:166-70.

45. Groenestege WM, Thebault S, van der Wijst J, van den Berg D, Janssen R, Tejpar S, et al. Impaired basolateral sorting of pro-EGF causes isolated recessive renal hypomagnesemia. J Clin Invest. 2007;117:2260-7.

46. Dimke H, Monnens L, Hoenderop JG, Bindels RJ. Evaluation of hypomagnesemia: lessons from disorders of tubular transport. Am J Kidney Dis. 2013;62:377-83.

47. Chubanov V, Gudermann T. Trpm6. Handb Exp Pharmacol. 2014;222:503-20.

48. Chung KY, Shia J, Kemeny NE, Shah M, Schwartz GK, Tse A, et al. Cetuximab shows activity in colorectal cancer patients with tumors that do not express the epidermal growth factor receptor by immunohistochemistry. J Clin Oncol. 2005;23:1803-10.

49. da Cunha Santos G, Shepherd FA, Tsao MS. EGFR mutations and lung cancer. Annu Rev Pathol. 2011;6:49-69.

50. Chen P WL, Li H, Liu B,Zou Z. Incidence and risk of hypomagnesemia in advanced cancer patients treated with cetuximab: a metaanalysis. Oncol Lett. 2013;1915-20. 